Smith, Martin M. ORCID:

https://orcid.org/0000-0002-4754-3032, Saklofske, Donald H., Stoeber, Joachim and Sherry, Simon B. (2016) The Big Three Perfectionism Scale. Journal of Psychoeducational Assessment, 34 (7). pp. 670-687.

Downloaded from: http://ray.yorksj.ac.uk/id/eprint/2922/

The version presented here may differ from the published version or version of record. If you intend to cite from the work you are advised to consult the publisher's version: https://doi.org/10.1177/0734282916651539

Research at York St John (RaY) is an institutional repository. It supports the principles of open access by making the research outputs of the University available in digital form. Copyright of the items stored in RaY reside with the authors and/or other copyright owners. Users may access full text items free of charge, and may download a copy for private study or non-commercial research. For further reuse terms, see licence terms governing individual outputs. Institutional Repository Policy Statement

\title{
RaY
}

Research at the University of York St John

For more information please contact RaY at ray@yorksj.ac.uk 
The Big Three Perfectionism Scale: A New Measure of Perfectionism

Martin M. Smith and Donald H. Saklofske

University of Western Ontario

Joachim Stoeber

University of Kent

Simon B. Sherry

Dalhousie University

Accepted May 3, 2016 at Journal of Psychoeducational Assessment

Author Note

Martin M. Smith and Donald Saklofske, Department of Psychology, University of Western Ontario; Joachim Stoeber, School of Psychology, University of Kent; Simon Sherry, Department of Psychology and Neuroscience, Dalhousie University. This study was supported by the University of Western Ontario Faculty of Social Science Research Development Fund awarded to the second author. Correspondence cornering this article should be addressed to Martin M. Smith, Department of Psychology, University of Western Ontario, London N6A 5C2, Canada. E-mail: msmit454@uwo.ca. 


\begin{abstract}
This article introduces a new measure of dispositional perfectionism: the Big Three Perfectionism Scale (BTPS). The BTPS assesses three higher-order global factors (rigid perfectionism, self-critical perfectionism, narcissistic perfectionism) via 10 lower-order perfectionism facets (self-oriented perfectionism, self-worth contingencies, concern over mistakes, doubts about actions, self-criticism, socially prescribed perfectionism, other-oriented perfectionism, hypercriticism, grandiosity, entitlement). The present investigation examined the structure of the BTPS using exploratory factor analysis in Study 1 (288 undergraduates), and confirmatory factor analyses in Study 2 (352 community adults) and Study 3 (290 undergraduates). Additionally, in Study 3 the relationships among the BTPS, other measures of perfectionism, and the five-factor model of personality were investigated. Overall, findings provide first evidence for the reliability and validity of the BTPS as a multidimensional measure of perfectionism.
\end{abstract}

Keywords: perfectionism, rigidity, self-criticism, narcissism, factor analysis, reliability, validity, personality, gender 
The Big Three Perfectionism Scale:

A New Measure of Perfectionism

Perfectionism is a personality trait characterized by striving for flawlessness and setting excessively high standards for performance accompanied by overly critical evaluations of one's behavior (Frost, Marten, Lahart, \& Rosenblate, 1990; Hewitt \& Flett, 1991). Over the past 25 years, a wealth of evidence suggests two higher-order factors underlie and account for shared variance amongst lower-order perfectionism facets: personal standards perfectionism and evaluative concerns perfectionism (Dunkley, Blankstein, Halsall, Williams, \& Winkworth, 2000; Dunkley, Blankstein, Masheb, \& Grilo, 2006; Sherry, Gautreau, Mushquash, Sherry, \& Allen, 2014). Personal standards perfectionism involves a family of traits encompassing the tendency to demand perfection of oneself (i.e., self-oriented perfectionism; Hewitt \& Flett, 1991) and the propensity to hold unrealistically high personal expectations (i.e., personal standards; Frost et al., 1990). Evaluative concerns perfectionism involves a constellation of traits comprising the tendency to perceive others as demanding perfection (i.e., socially prescribed perfectionism; Hewitt \& Flett, 1991), have overly negative reactions to perceived failures and setbacks (i.e., concerns over mistakes; Frost et al., 1990), and doubts about performance abilities (i.e., doubts about actions; Frost et al., 1990).

Personal standards perfectionism is a double-edged form of perfectionism. On the one hand, personal standards perfectionism is associated with negative characteristics, processes, and outcomes such as neuroticism, ruminative brooding, and depression (Smith, Sherry, Rnic, Saklofske, Enns, \& Gralnick, 2016; see Hewitt \& Flett, 2004, for a review). On the other hand, personal standards perfectionism has been shown to be associated with positive characteristics such as conscientiousness and task-oriented coping (Dunkley \& Blankstein, 2000; Rice, Ashby, $\&$ Slaney, 2007). In contrast, evaluative concerns perfectionism clearly represents a negative 
form of perfectionism showing strong and consistent negative associations with negative affect and various indicators of psychological maladjustment (see Stoeber \& Otto, 2006, for a review).

Typically, personal standards perfectionism and evaluative concerns perfectionism are assessed by combining subscales from the two most prominent and widely used measures of multidimensional perfectionism: the Multidimensional Perfectionism Scales by Frost et al. (FMPS; 1990) and Hewitt and Flett (HF-MPS; 1991). While useful, a shortcoming of this patchwork approach reflects not so much a particular limitation of the FMPS or HF-MPS, but rather a fundamental scientific principle: as knowledge of a construct advances, the constructs definition must be revisited (Smith, Fischer, \& Fister, 2003).

Following an extensive literature review, 10 facets appeared worthy of inclusion in a contemporary perfectionism measure resulting in the development of scales labeled self-oriented perfectionism, self-worth contingencies, concern over mistakes, doubts about actions, selfcriticism, socially-prescribed perfectionism, other-oriented perfectionism, hypercriticism, entitlement, and grandiosity. Self-worth contingencies were included in consideration of research suggesting that disentangling self-oriented perfectionism from self-worth contingencies provides a more detailed and informative assessment of personal standards perfectionism (DiBartolo, Frost, Chang, LaSota, \& Grills, 2004). Self-criticism reflected evidence suggesting that selfcriticism is a core component of perfectionism (e.g., Blankstein \& Dunkley, 2002; Dunkley, Zuroff, \& Blankstein, 2003). Additionally, to allow for the assessment of a third superordinate factor labeled narcissistic perfectionism (see Nealis, Sherry, Sherry, Stewart, \& MacNeil, 2015) four scales were included: other-oriented perfectionism, hypercriticism, entitlement, and grandiosity. This new measure is named the Big Three Perfectionism Scale (BTPS).

\section{The Big Three Perfectionism Scale}

The BTPS was developed to provide a fine-grained analysis of multidimensional 
perfectionism. To this aim, the authors constructed a 45-item self-report questionnaire designed to measure three global perfectionism factors (rigid perfectionism, self-critical perfectionism, and narcissistic perfectionism), comprised of 10 core perfectionism facets. Scales constructed using facets assuage theoretical confusion, reduce the possibility of omitting core content, and afford greater reliability and precision in assessment (Comrey, 1988; Paunonen, Haddock, Fosterling, \& Keinonen, 2003; Reise, Waller, \& Comrey, 2000; Smith, Fister, \& Fischer, 2003). The BTPS's first global factor is labeled rigid perfectionism. Whereas the label was inspired by the subscale of the same name from the Personality Inventory for DSM-5 (Krueger, Derringer, Markon, Watson, \& Skodol, 2013; see Stoeber, 2014), the items were specifically written to capture the rigid insistence that one's own performance must be flawless, perfect, and without errors. Rigid perfectionism borrows heavily from the work of DiBartolo et al. (2004), Hewitt and Flett (1991), Sturman, Flett, Hewitt, and Rudolph (2009), and Stoeber and Childs (2010), and is comprised of two facets: self-oriented perfectionism and self-worth contingencies. Self-oriented perfectionism refers to the belief that striving for perfection, as well as being perfect, are important (Hewitt \& Flett, 1991; Stoeber \& Childs, 2010). Self-worth contingencies refer to the tendency to base self-worth on self-imposed perfectionistic standards (DiBartolo et al., 2004; Sturman et al., 2009).

The BTPS's second global factor is self-critical perfectionism. We operationalized selfcritical perfectionism following the model proposed by Dunkley, Zuroff, and Blankstein (2003) in which self-critical perfectionism subsumes four facets: concern over mistakes, doubts about actions, self-criticism, and socially prescribed perfectionism. Concern over mistakes is the tendency to have overly negative reactions to perceived setbacks and failures (Frost et al., 1990). Doubts about actions reflects uncertainties about performance (Frost et al., 1990). Self-criticism measures the tendency to engage in harsh self-criticism when performance falls short of 
perfection (Dunkley, Zuroff, \& Blankstein, 2003). Socially prescribed perfectionism denotes a tendency to perceive others as demanding perfection (Hewitt \& Flett, 1991).

The third BTPS global factor is narcissistic perfectionism. Narcissistic perfectionism was operationalized following Nealis et al.'s (2015) model and is comprised of four facets: otheroriented perfectionism, hypercriticism, entitlement, and grandiosity. Other-oriented perfectionism is the tendency to hold unrealistic expectations for others (Hewitt \& Flett, 1991). Hypercriticism involves harsh devaluation of others and their imperfections (Nealis et al., 2015). Entitlement refers to the belief that one is entitled to perfect or special treatment (Nealis et al., 2015). Grandiosity denotes a sustained view of oneself as perfect or superior to others (Flett, Sherry, Hewitt, \& Nepon, 2014; Nealis, Sherry, Lee-Baggley, Stewart, \& Macneil, in press; Stoeber, Sherry, \& Nealis, 2015). While narcissistic perfectionism is often discussed in theory (e.g., Beck, Davis, \& Freeman, 2014; Hewitt \& Flett, 1991; Millon, Grossman, Millon, Meagher, \& Ramnath, 2004), the BTPS offers the only self-report measure designed specifically to assess individuals who believe they are perfect, superior to others, and justified in holding unrealistic expectations (i.e., narcissistic perfectionists).

Additionally, narcissistic perfectionism, as operationalized in the BTPS, is distinguishable from currently available measures of narcissism given that other oriented perfectionism (e.g., "I expect those close to me to be perfect"), hyper-criticism (e.g., "I get frustrated when other people make mistakes"), entitlement (e.g., "It bothers me when people don't notice how perfect I am"), and grandiosity (e.g., "I know that I am perfect") directly reference either perfection or highly related concepts (e.g., concern over others mistakes). Moreover, narcissistic perfectionists tend not to perceive a discrepancy between the actual and ideal self which likely fosters a sense of grandiose entitlement and potentiates excessive criticism of others. 


\section{The Present Research}

Against this background, the aim of the present research was to provide a first investigation of the reliability and validity of the newly constructed BTPS across two university student samples and one community adult sample. Exploratory and confirmatory factor analyses were conducted to evaluate the homogeneity of the BTPS facets, the anticipated higher-order three-factor solution, and gender invariance. Additionally, to examine convergent and differential validity, correlations with established measures of multidimensional perfectionism and the five-factor model of personality (Cost \& McCrae, 2008) were examined.

\section{Data Analytic Strategy}

In Study 1, exploratory factor analysis was conducted using SPSS 22. Following the recommendations of Fabrigar, Wegener, MacCallum, and Strahan (1999), we used maximum likelihood estimation with oblique rotation (promax) for factor extraction. Parallel analysis was used to determine the number of factors to retain (Fabrigar et al., 1999). In Study 2 and Study 3, confirmatory factor analysis (CFA) was conducted using weighted least squares estimation (WLSMV) in Mplus 7.2 (Muthén \& Muthén, 2013). Samejima’s (1969) graded response models were produced by specifying factor indicators as ordered categorical variables. WLSMV with

mean and variance adjusted $\chi^{2}$ tests were used for model comparison. In addition to WLSMV $\chi^{2}$, we used the following fit indices for model evaluation: root mean square error of approximation (RMSEA), comparative fit index (CFI), and Tucker-Lewis index (TLI). Following Hu and Bentler (1998), we considered RMSEA values close to .06 as indicative of good fit, values between .07 and .08 as indicative of moderate fit, values between .08 and .10 as indicative of marginal fit, and values greater than .10 as indicative of poor fit. Regarding CFI and TLI, values in the range of .95 or above suggest good model fit and values between .90 and .95 suggest marginally acceptable fit. 


\section{Data Screening}

Investigating whether any participants gave uniform responses resulted in the exclusion of six participants from Study 1 and seven participants from Study 2 who showed zero variance across all 45 BTPS items. Next we computed the scores for each of the 10 BTPS facets. Because multivariate outliers can severely distort the results of factor analyses (Tabachnick \& Fidell, 2007), we excluded one participant from Study 1, nine participants from Study 2, and one participant from Study 3 who showed a Mahalanobis distance larger than the critical value of $\chi^{2}(10)=29.59, p<.001$. With this, Study 1 comprised 288 university students, Study 2 comprised 352 community adults, and Study 3 comprised 290 university students.

\section{Study 1: Item Selection, Reduction, and Exploratory Analysis}

The first step in developing a new self-report measure involves meticulous explication of the target construct, rational generation of a large item pool, and the selection of the best items (Jackson, 1975). The purpose of Study 1 was thus to develop a set of homogenous facets, derived from theory and research, that measure three global factors of perfectionism (rigid, self-critical, and narcissistic perfectionism) via 10 core perfectionism facets. An initial pool of 102 items was generated by the authors over the course of a year. Items judged as problematic (e.g., overly redundant or ambiguous) were deleted. This resulted in the 45-item Big Three Perfectionism Scale (BTPS; see Supplementary Material).

\section{Method}

\section{Participants}

Participants were 288 undergraduates (199 men, 89 women) mostly in their first year of study $(88.1 \%)$ recruited from the first author's university. Self-reported ethnicities were White (51.2\%), Chinese (14.6\%), South Asian (6.8\%), Korean (3.7\%), multiracial (8.5\%), and other (9.8\%) with 5.4\% missing. 


\section{Measures and Procedure}

Participants were administered the 45-item BTPS with instructions to respond to each item using a 5-point rating scale from 1 (strongly disagree) to 5 (strongly agree). The arrangement of items was randomized.

\section{Results}

\section{Descriptive Statistics}

The means, standard deviations, bivariate correlations, and internal consistency of the BTPS facets and global factors are shown in Table 1. Internal consistency measured by Cronbach's alpha ranged from .79 to .89 for the 10 facets and from .92 to .93 for the three global factors. In contrast to the facets comprising other factors, the means for the Narcissistic Perfectionism facets were low due to a lower frequency of endorsement. Bivariate correlations complemented prior research (e.g., Nealis et al., 2015) and indicated that narcissistic perfectionism and self-critical perfectionism are conceptually, as well as empirically, distinguishable.

\section{Exploratory Analysis of BTPS Items and Facets}

The Kaiser-Meyer-Olkin measure of sampling adequacy was .93 indicating that the data

were suitable for factor analysis. Separate exploratory factor analyses were performed on each of the 10 facets (see Table 2). We also conducted exploratory factor analysis using all 45 BTPS items. Parallel analysis indicated that four factors were significant and should be retained (cf. Preacher \& MacCallum, 2003): Factor 1 had an eigenvalue of 12.89 (29.26\% of the variance), Factor 2 had an eigenvalue of 5.99 (13.93\% of the variance), Factor 3 had an eigenvalue of 3.04 (7.07\% of the variance), and Factor 4 had an eigenvalue of 1.87 (4.34\% of the variance). As the fourth factor contained no salient loadings, a three-factor solution was forced and loadings are shown in Table 3. Furthermore, a second-order exploratory factor analysis was conducted (see 
Table 4).

\section{Discussion}

Results supported the unidimensionality and homogeneity (Comrey, 1988) of each of the 10 BTPS facets (see Table 1). Results also indicated that the BTPS facets and global factors had adequate internal consistency. Additionally, results suggested that the BTPS is comprised of three higher-order factors corresponding to the three proposed global factors (rigid, self-critical, and narcissistic perfectionism) that in turn underlie 10 lower-order perfectionism facets (selforiented perfectionism, self-worth contingencies, concern over mistakes, doubts about actions, self-criticism, socially prescribed perfectionism, other-oriented perfectionism, hypercriticism, entitlement, and grandiosity). In light of findings that self-worth contingencies are central to understanding the link between perfectionism and psychological maladjustment (DiBartolo et al., 2004; Sturman et al., 2009), the inclusion of self-worth contingencies as a facet of perfectionism is a notable strength of the BTPS.

\section{Study 2: Confirmatory Factor Analysis}

When creating a new instrument, it is important to evaluate its underlying structure across different groups. It is also crucial to determine the extent to which the proposed structure is invariant across men and women (Reise, Waller, \& Comrey, 2000). Additionally, as noted by Smith et al. (2003), the theoretical contention that broad factors underlie groups of facets must be empirically tested, rather than assumed.

\section{Method}

\section{Measures and Procedure}

The 45-item BTPS was administered to 367 community adults (178 men, 174 women, 15 not reported) recruited from CrowdFlower, an internet platform comparable to Amazon's Mechanical Turk which is used to obtain reliable data from community samples (e.g., 
Buhrmester, Kwang, \& Gosling, 2011). Participants were asked to indicate their agreement to the BTPS items using a 5-point scale ranging from (1) strongly disagree to (5) strongly agree.

Country of origin was restricted to the United States and Canada. Participants were paid a small fee (\$1.00). Self-reported ethnicities were as follows: White (81.7\%), Latin American (6.0\%), Chinese (3.0\%), multiracial (1.9\%), and other (3.9\%) with $3.5 \%$ missing.

\section{Descriptive Statistics}

The means, standard deviations, bivariate correlations, and internal consistency of the BTPS facets and global factors are presented in Table 1. Cronbach's alpha ranged from .83 to .90 for the 10 facets and from .92 to .93 for the three global factors.

\section{Confirmatory Factor Analysis of the BTPS Facets}

To evaluate the homogeneity of the 10 BTPS facets, we assessed the unidimensionality of each facet separately using CFA. Loadings ranged from .72 to .95 (see Table 5). Additionally, when all 10 BTPS facets were estimated simultaneously, model fit was good: WLSMV $\chi^{2}(900)$ $=1767.64, \mathrm{RMSEA}=.051(90 \%$ confidence interval $[\mathrm{CI}]=.048-.055), \mathrm{CFI}=.971$, and $\mathrm{TLI}=$ 968.

\section{Second-Order Confirmatory Factor Analysis}

To further examine the relationship between the 10 BTPS facets we compared the fit of models with one, two, and three higher-order global factors. For the one-factor model, all 10 BTPS facets were specified to load on a single global factor. For the two-factor model, selforiented perfectionism and self-worth contingencies were specified to load on one global factor, and all remaining facets were specified to load on a second global factor. Finally, for the threefactor model, self-oriented perfectionism and self-worth contingencies were specified to load on one global factor (rigid perfectionism), concern over mistakes, doubts about actions, and selfcriticism, and socially-prescribed perfectionism to load on a second global factor (self-critical 
perfectionism), and other-oriented perfectionism, hypercriticism, entitlement, and grandiosity to load on a third global factor (narcissistic perfectionism).

The fit of the model with one second-order global factor was poor: WLSMV $\chi^{2}(935)=$ 4052.27, RMSEA $=.095(90 \% \mathrm{CI}=.092-.098), \mathrm{CFI}=.896$, and TLI $=.890$. In contrast, the fit of the model with two global factors was only marginally acceptable: WLSMV $\chi^{2}(934)=$ 3780.28, $\mathrm{RMSEA}=.091(90 \% \mathrm{CI}=.088-.094), \mathrm{CFI}=.905$, and $\mathrm{TLI}=.899$. As expected, the fit of the model (see Figure 1) with three global factors was good: WLSMV $\chi^{2}(932)=2463.50$, RMSEA $=.067(90 \% \mathrm{CI}=.064-.070), \mathrm{CFI}=.949$, and TLI $=.946$. Furthermore, the model with three global factors fit significantly better than the model with only two: WLSMV $\Delta \chi^{2}(2)=$ $159.73, p<.001$.

\section{Multiple-Group CFA for Invariance Across Gender}

Measurement invariance across men and women was investigated. The fit of the configural model was marginally acceptable: WLSMV $\chi^{2}(1884)=3037.78, \operatorname{RMSEA}=.066$ $(90 \% \mathrm{CI}=.061-.070), \mathrm{CFI}=.913$, and $\mathrm{TLI}=.908$. Constraining factor loadings to be equal across men and women did not result in a significant loss of fit (WLSMV $\Delta \chi^{2}[43]=47.00, p=$ .312). Similarly, constraining item thresholds, as well as factor loadings, to be equal across men and women did not result in a significant loss of fit (WLSMV $\left.\Delta \chi^{2}[32]=155.08, p=.083\right)$.

\section{Discussion}

Study 2 provided further evidence that each of the 10 BTPS facets is reliable and homogenous. Results also supported the multidimensional nature of the BTPS and suggest it is best conceptualized as measuring three oblique global factors that underlie 10 perfectionism facets. Additionally, the BTPS appeared to show the same factor structure in men and women.

\section{Study 3: Convergent and Divergent Validity}


In Study 3, convergent validity was assessed by administering the two most prominent and widely used measures of perfectionism — the Frost Multidimensional Perfectionism Scale (FMPS; Frost et al., 1990) and the Hewitt-Flett Multidimensional Perfectionism Scale (HF-MPS; Hewitt \& Flett, 1991) —alongside a measure of the five-factor model of personality. We expected that after controlling for variance attributable to self-critical perfectionism and narcissistic perfectionism that rigid perfectionism would show a distinct positive relation with conscientiousness (Hill, McIntire, \& Bacharach, 1997; Rice, Ashby, \& Slaney, 2007). Similarly, we expected that self-critical perfectionism would have a unique positive relation with neuroticism (Bekes et al., 2015) and narcissistic perfectionism would have a distinct negative relation with agreeableness (Nealis et al., 2015). Given the importance of replication, the factor structure of the BTPS was re-evaluated.

\section{Method}

\section{Participants and Procedure}

The sample was comprised of 290 undergraduates ( 88 men, 202 women) recruited from the first author's university. Participants completed the BTPS, the FMPS, the HF-MPS, and Saucier's (1994) Big-Five Mini-Markers. Self-reported ethnicities were as follows: White (53.0\%), Chinese (21.6\%), South Asian (7.7\%), Korean (2.4\%), Arab (1.4\%), and other (4.8\%) with $9.1 \%$ missing.

\section{Confirmatory Factor Analysis of the BTPS Facets}

The unidimensionality of each facet was investigated using CFA (see Table 5). When all 10 facet scales were simultaneously estimated, model fit was again good: WLSMV $\chi^{2}(900)=$ 1165.44, $\mathrm{RMSEA}=.032(90 \% \mathrm{CI}=.027-.037), \mathrm{CFI}=.981$, and $\mathrm{TLI}=.979$.

\section{Second-Order Confirmatory Factor Analysis}

As in Study 2, the fit of the model with 10 first-order facets and one second-order global 
factor was poor: $\operatorname{WLSMV} \chi^{2}(935)=4145.73, \mathrm{RMSEA}=.109(90 \% \mathrm{CI}=.106, .113), \mathrm{CFI}=.765$, and TLI $=.752$. Similarly, the fit of the model with two global factors was poor: WLSMV $\chi^{2}$ $(934)=3647.76, \mathrm{RMSEA}=.101(90 \% \mathrm{CI}=.097, .104), \mathrm{CFI}=.802$, and $\mathrm{TLI}=.790$. In contrast, as was expected, the fit of the model with three global factors was good (see Figure 2): WLSMV $\chi^{2}(932)=1627.79, \mathrm{RMSEA}=.051(90 \% \mathrm{CI}=.047, .055), \mathrm{CFI}=.949$, and $\mathrm{TLI}=.946$.

Moreover, the model with three global factors fit significantly better than the model with two global factors: WLSMV $\Delta \chi^{2}(2)=184.64, p<.001$.

\section{Correlations with Perfectionism and Personality Indicators}

Correlations between the 10 BTPS facets with other measures of perfectionism and the five-factor model of personality are presented in Table 6. Bivariate correlations and semipartial correlations between the BTPS's three global factors (rigid, self-critical, and narcissistic perfectionism) and indicators of perfectionism and personality are presented in Table 7. All patterns of significant correlations were as expected. Following Cohen's (1992) guidance of what constitutes large, medium, and small effects, rigid perfectionism showed a large-sized positive correlation with personal standards and self-oriented perfectionism, a medium-sized positive correlation with conscientiousness, and a small-sized positive correlation with neuroticism. Self-critical perfectionism showed large-sized positive correlations with concern over mistakes, doubts about actions, self-oriented perfectionism, and socially prescribed perfectionism, a medium-sized positive correlation with neuroticism, a medium-sized negative correlation with extraversion, and a small-sized negative correlation with agreeableness. Narcissistic perfectionism showed a large-sized positive correlation with other-oriented perfectionism, a large-sized negative correlation with agreeableness, and a medium-sized positive correlation with neuroticism. Examining the semipartial correlations revealed that after controlling for rigid perfectionism and narcissistic perfectionism, self-critical perfectionism was 
the only global factor significantly positively correlated with doubts about actions, parental criticism, and socially prescribed perfectionism.

\section{General Discussion}

The aim of this research was to present validity and reliability evidence regarding a new measure of multidimensional perfectionism: the Big Three Perfectionism Scale (BTPS). Across two university samples and one community adult sample, each of the 10 BTPS facets (selforiented perfectionism, self-worth contingencies, concern over mistakes, doubts about actions, self-criticism, socially prescribed perfectionism, other-oriented perfectionism, hypercriticism, entitlement, and grandiosity) showed clear homogeneity and unidimensionality (Smith, Fischer, \& Fister, 2003). Additionally, both exploratory and confirmatory factor analyses indicated that the majority of common variance amongst the BTPS's 10 core facets was accounted for by three higher-order global factors: rigid perfectionism, self-critical perfectionism, and narcissistic perfectionism. Whereas moderate to large correlations were observed among the three global factors of the BTPS across the three samples, our factor analytic findings suggest the BTPS is best conceptualized as multidimensional. Results also suggested that the BTPS shows the same factorial structure in men and women. Bivariate and semipartial correlations were in line with expectations and provided further evidence regarding the validity of the BTPS. Rigid perfectionism had a distinct association with conscientiousness, self-critical perfectionism had distinct association with neuroticism, and narcissistic perfectionism had a distinct association with agreeableness.

Relative to existing measures, the BTPS has a number of notable features. In particular, the BTPS differentiates self-oriented perfectionism from self-worth contingencies, and by doing so, allows for a more detailed and informative assessment of multidimensional perfectionism (see Stoeber \& Childs, 2010). Moreover, the BTPS offers the only self-report measure capable of 
assessing individuals who believe they are perfect, superior to others, and justified in holding unrealistic expectations (i.e., narcissistic perfectionists). Furthermore, an important strength of the BTPS is that it provides researchers with the option of studying perfectionism at either its lowest level via the 10 BTPS facets or its broadest level via the three global perfectionism factors. Additionally, the BTPS is the only available instrument capable of assessing personal standards perfectionism, evaluative concerns perfectionism, and narcissistic perfectionism using a single self-report scale.

\section{Limitations and Future Directions}

The results of the present study should be considered in light of its limitations. The magnitude of intercorrelations amongst facets and global factors in the student and community samples were notably distinct (cf. Figures 1 and 2). A potential explanation is that intercorrelations between the BTPS factors may vary across samples due to the presence of a moderating factor. For example, in the student samples less than perfect grades may attenuate grandiosity and amplify self-criticism. Nonetheless, this speculation remains to be tested. Additionally, all samples were comprised of predominately Caucasian participants from Canada and the United States. Future research should investigate the generalizability of the BTPS across more ethnically diverse samples, as well as additional nationalities. Further research is also needed to determine how narcissistic perfectionism relates to grandiose and vulnerable narcissism (Stoeber et al., 2015). Moreover, in the present research validation measures were restricted to the FMPS, HF-MPS, and the five-factor model of personality. Future studies should investigate how the BTPS's global factors and facets relate to social desirability, alternative measure of perfectionism, as well as other models of personality (e.g., the HEXACO model; Ashton \& Lee, 2007). A fuller understanding of the incremental validity of the BTPS is also needed. Future research should determine if the BTPS predicts outcomes (e.g., academic 
performance or prosocial vs. antisocial behaviors) beyond existing measures such as the FMPS and the HFMPS. Additionally, a version of the BTPS that allows for collection of informant reports would help to overcome potential limitations associated with use of participant self-report (e.g., self-serving reporting biases; Stoeber \& Hotham, 2013).

\section{Concluding Remarks}

Following from the results of these preliminary studies, the BTPS appears to be a promising new instrument for the multidimensional assessment of the various facets of perfectionism and their superordinate factors (rigid perfectionism, self-critical perfectionism, and narcissistic perfectionism). Additionally, the BTPS provides the first and only scale comprising a measure of narcissistic perfectionism. 


\section{References}

Ashton, M. C., \& Lee, K. (2007). Empirical, theoretical, and practical advantages of the HEXACO model of personality structure. Personality and Social Psychology Review, 11, $150-166$.

Beck, A. T., Davis, D. D., \& Freeman, A. (2014). Cognitive therapy of personality disorders. New York: Guilford.

Bekes, V., Dunkley, D. M., Taylor, G., Zuroff, D. C., Lewkowski, M., Foley, J. E., . . . Westreich, R. (2015). Chronic stress and attenuated improvement in depression over 1 year: The moderating role of perfectionism. Behavior Therapy, 46, 478-492.

Blankstein, K. R., \& Dunkley, D. M. (2002). Evaluative concerns, self-critical, and personal standards perfectionism: A structural equation modeling strategy. In G. L. Flett, \& P. L. Hewitt (Eds.) Perfectionism: Theory, research, and treatment (pp. 285-315). Washington: American Psychological Association.

Buhrmester, M., Kwang, T., \& Gosling, S. D. (2011). Amazon's Mechanical Turk: A new source of inexpensive, yet high-quality, data? Perspectives on Psychological Science, 6, 3-5.

Cohen, J. (1992). A power primer. Psychological Bulletin, 112, 155-159.

Comrey, A. L. (1988). Factor-analytic methods of scale development in personality and clinical psychology. Journal of Consulting and Clinical Psychology, 56, 754-761.

Costa, P., \& McCrae, R. (2008). The revised NEO personality inventory (NEO-PI-R). In G. Boyle, G. Matthews, \& D. Saklofske (Eds.), The SAGE handbook of personality theory and assessment: Volume 2 - Personality measurement and testing. (pp. 179-199). London: Sage.

DiBartolo, P. M., Frost, R. O., Chang, P., LaSota, M., \& Grills, A. E. (2004). Shedding light on the relationship between personal standards and psychopathology: The case for 
contingent self-worth. Journal of Rational-Emotive \& Cognitive-Behavior Therapy, 22, 237-250.

Dunkley, D. M., Blankstein, K. R., Halsall, J., Williams, M., \& Winkworth, G. (2000). The relation between perfectionism and distress: Hassles, coping, and perceived social support as mediators and moderators. Journal of Counseling Psychology, 47, 437-453.

Dunkley, D. M., Blankstein, K. R., Masheb, R. M., \& Grilo, C. M. (2006). Personal standards and evaluative concerns dimensions of "clinical" perfectionism: A reply to Shafran et al. (2002, 2003) and Hewitt et al. (2003). Behaviour Research and Therapy, 44, 63-84.

Dunkley, D. M., Zuroff, D. C., \& Blankstein, K. R. (2003). Self-critical perfectionism and daily affect: Dispositional and situational influences on stress and coping. Journal of Personality and Social Psychology, 84, 234-252.

Fabrigar, L. R., Wegener, D. T., MacCallum, R. C., \& Strahan, E. J. (1999). Evaluating the use of exploratory factor analysis in psychological research. Psychological Methods, 4, 272299.

Flett, G. L., Sherry, S. B., Hewitt, P. L., \& Nepon, T. (2014). Understanding the narcissistic perfectionists among us: Grandiosity, vulnerability, and the quest for the perfect self. In A. Besser (Ed.), Handbook of psychology of narcissism: Diverse perspectives (pp. 4366). New York: Nova Science Publishers.

Frost, R. O., Marten, P., Lahart, C., \& Rosenblate, R. (1990). The dimensions of perfectionism. Cognitive Therapy and Research, 14, 449-468.

Hewitt, P. L., \& Flett, G. L. (1991). Perfectionism in the self and social contexts: Conceptualization, assessment, and association with psychopathology. Journal of Personality and Social Psychology, 60, 456-470.

Hewitt, P. L., \& Flett, G. L. (2004). Multidimensional Perfectionism Scale (MPS): Technical 
manual. Toronto: Multi-Health Systems.

Hill, R. W., McIntire, K., \& Bacharach, V. R. (1997). Perfectionism and the Big Five factors. Journal of Social Behavior \& Personality, 12, 257-270.

Hu, L., \& Bentler, P. M. (1998). Fit indices in covariance structure modeling: Sensitivity to underparameterized model misspecification. Psychological Methods, 3, 424-453.

Jackson, D. N. (1975). The relative validity of scales prepared by naive item writers and those based on empirical methods of personality scale construction. Educational and Psychological Measurement, 35, 361-370.

Krueger, R. F., Derringer, J., Markon, K. E., Watson, D., \& Skodol, A. E. (2013). The Personality Inventory for DSM-5 (PID-5). Washington, DC: American Psychiatric Association.

Millon, T., Grossman, S., Millon, C., Meagher, S., \& Ramnath, R. (2004). Personality disorders in modern life (2nd ed). Hoboken, NJ: Wiley.

Muthén, L., \& Muthén, B. O. (2013). Mplus user’s guide. Los Angeles, CA: Muthén \& Muthén.

Nealis, L. J., Sherry, S. B., Lee-Baggley, D. L., Stewart, S. H., \& Macneil, M. A. (in press).

Revitalizing narcissistic perfectionism: Evidence of reliability and validity of an emerging construct. Journal of Psychopathology and Behavioral Assessment.

Nealis, L. J., Sherry, S. B., Sherry, D. L., Stewart, S. H., \& Macneil, M. A. (2015). Towards a better understanding of narcissistic perfectionism: Evidence of factorial validity, incremental validity, and mediating mechanisms. Journal of Research in Personality, 57, $11-25$.

Paunonen, S. V., Haddock, G., Forsterling, F., \& Keinonen, M. (2003). Broad versus narrow personality measures and the prediction of behaviour across cultures. European Journal of Personality, 17, 413-433. 
Preacher, K. J., \& MacCallum, R. C. (2003). Repairing Tom Swift's electric factor analysis machine. Understanding Statistics, 2, 13-43.

Reise, S. P., Waller, N. G., \& Comrey, A. L. (2000). Factor analysis and scale revision. Psychological Assessment, 12, 287-297.

Rice, K. G., Ashby, J. S., \& Slaney, R. B. (2007). Perfectionism and the five-factor model of personality. Assessment, 14, 385-398.

Samejima, F. (1969). Estimation of latent ability using a response pattern of graded scores. Psychometrika Monograph Supplement, 34, 100.

Saucier, G. (1994). Mini-Markers: A brief version of Goldberg's unipolar Big-Five markers. Journal of Personality Assessment, 63, 506-516.

Sherry, S. B., Gautreau, C. M., Mushquash, A. R., Sherry, D. L., \& Allen, S. L. (2014). Selfcritical perfectionism confers vulnerability to depression after controlling for neuroticism: A longitudinal study of middle-aged, community-dwelling women Personality and Individual Differences, 69, 1-4.

Smith, G. T., Fischer, S., \& Fister, S. M. (2003). Incremental validity principles in test construction. Psychological Assessment, 15, 467-477.

Smith, M. M., Sherry, S. B., Rnick, K., Saklofske, D. H., Enns, M. W., \& Gralnick, T. M. (2016). Are perfectionism dimensions vulnerability factors for depressive symptoms after controlling for neuroticism? A meta-analysis of 10 longitudinal studies. European Journal of Personality, 30, 201-212.

Stoeber, J. (2014). Multidimensional perfectionism and the DSM-5 personality traits. Personality and Individual Differences, 64, 115-120.

Stoeber, J., \& Childs, J. H. (2010). The assessment of self-oriented and socially prescribed perfectionism: Subscales make a difference. Journal of Personality Assessment, 92, 577- 
585.

Stoeber, J., \& Hotham, S. (2013). Perfectionism and social desirability: Students report increased perfectionism to create a positive impression. Personality and Individual Differences, 55, 626-629.

Stoeber, J., \& Otto, K. (2006). Positive conceptions of perfectionism: Approaches, evidence, challenges. Personality and Social Psychology Review, 10, 295-319.

Stoeber, J., Sherry, S. B., \& Nealis, L. J. (2015). Multidimensional perfectionism and narcissism: Grandiose or vulnerable? Personality and Individual Differences, 80, 85-90.

Sturman, E. D., Flett, G. L., Hewitt, P. L., \& Rudolph, S. G. (2009). Dimensions of perfectionism and self-worth contingencies in depression. Journal of Rational-Emotive \& Cognitive-Behavior Therapy, 27, 213-231.

Tabachnick, B. G., \& Fidell, L. S. (2007). Using Multivariate Statistics (5th ed.). Boston, MA: Pearson. 


\section{BIG THREE PERFECTIONISM SCALE}

Table 1

Big Three Perfectionism Scale (BTPS): Bivariate correlations, means, standard deviations, and Cronbach's alphas

\begin{tabular}{|c|c|c|c|c|c|c|c|c|c|c|c|c|c|c|c|c|}
\hline Scale. & 1 & 2 & 3 & 4 & 5 & 6 & 7 & 8 & 9 & 10 & 11 & 12 & 13 & $M$ & $S D$ & $\alpha$ \\
\hline \multicolumn{17}{|l|}{ Facets } \\
\hline 1. Self-oriented perfectionism & - & $.87^{* * *}$ & $.62^{* * *}$ & $.47^{* * *}$ & $.73^{* * *}$ & $.57^{* * *}$ & $.55^{* * *}$ & $.67^{* * *}$ & $.57^{* * *}$ & $.61^{* * *}$ & $.97^{* * *}$ & $.69^{* * *}$ & $.66^{* * *}$ & 2.87 & 0.98 & .89 \\
\hline 2. Self-worth contingencies & $.84^{* * *}$ & - & $.66^{* * *}$ & $.52^{* * *}$ & $.74^{* * *}$ & $.59^{* * *}$ & $.59^{* * *}$ & $.69^{* * *}$ & $.63^{* * *}$ & $.61^{* * *}$ & $.97^{* * *}$ & $.69^{* * *}$ & $.66^{* * *}$ & 2.75 & 0.96 & .88 \\
\hline 3. Concern over mistakes & $.47^{* * *}$ & $.56^{* * *}$ & - & $.74^{* * *}$ & $.83^{* * *}$ & $.46^{* * *}$ & $.54^{* * *}$ & $.50^{* * *}$ & $.46^{* * *}$ & $.36^{* * *}$ & $.66^{* * *}$ & $.92^{* * *}$ & $.51^{* * *}$ & 2.94 & 0.99 & .88 \\
\hline 4. Doubts about actions & $.30^{* * *}$ & $.35^{* * *}$ & $.63^{* * *}$ & - & $.67^{* * *}$ & $.34^{* * *}$ & $.42^{* * *}$ & $.42^{* * *}$ & $.40^{* * *}$ & $.27^{* * *}$ & $.51^{* * *}$ & $.87^{* * *}$ & $.42^{* * *}$ & 2.84 & 1.00 & .90 \\
\hline 5. Self-criticism & $.57^{* * *}$ & $.65^{* * *}$ & $.79^{* * *}$ & $.56^{* * *}$ & - & $.47^{* * *}$ & $.55^{* * *}$ & $.58^{* * *}$ & $.50^{* * *}$ & $.46^{* * *}$ & $.76^{* * *}$ & $.89^{* * *}$ & $.58^{* * *}$ & 2.86 & 1.03 & .89 \\
\hline 6. Socially-prescribed perfectionism & $.31^{* * *}$ & $.33^{* * *}$ & $.54^{* * *}$ & $.52^{* * *}$ & $.57^{* * *}$ & - & $.55^{* * *}$ & $.61^{* * *}$ & $.56^{* * *}$ & $.56^{* * *}$ & $.60^{* * *}$ & $.74^{* * *}$ & $.63^{* * *}$ & 2.63 & 0.91 & .83 \\
\hline 7. Hypercriticism & $.35^{* * *}$ & $.33^{* * *}$ & $.25^{* * *}$ & $.14^{*}$ & $.30^{* * *}$ & $.23^{* *}$ & - & $.76^{* * *}$ & $.76^{* * *}$ & $.72^{* * *}$ & $.59^{* * *}$ & $.59^{* * *}$ & $.89^{* * *}$ & 2.42 & 0.92 & .83 \\
\hline 8. Other-oriented perfectionism & $.32^{* * *}$ & $.31^{* * *}$ & $.19^{* *}$ & .06 & $.27^{* * *}$ & $.36^{* *}$ & $.71^{* * *}$ & - & $.78^{* * *}$ & $.76^{* * *}$ & $.70^{* * *}$ & $.60^{* * *}$ & $.92^{* * *}$ & 2.81 & 0.94 & .90 \\
\hline 9. Entitlement & $.27^{* * *}$ & $.28^{* * *}$ & $.18^{* *}$ & .07 & $.25^{* * *}$ & $.34^{* * *}$ & $.58^{* * *}$ & $.72^{* * *}$ & - & $.81^{*}$ & $.62^{* * *}$ & $.55^{*}$ & $.92^{* * *}$ & 2.15 & 1.01 & .89 \\
\hline 10. Grandiosity & $.36^{* * *}$ & $.28^{* * *}$ & .08 & .06 & $.21^{* * *}$ & $.25^{* * *}$ & $.56^{* * *}$ & $.63^{* * *}$ & $.69^{* * *}$ & - & $.63^{* * *}$ & $.55^{* * *}$ & $.92^{* * *}$ & 2.28 & 0.99 & .86 \\
\hline \multicolumn{17}{|l|}{ Global factors } \\
\hline 11. Rigid perfectionism & $.96^{* * *}$ & $.96^{* * *}$ & $.53^{*}$ & $.34^{* * *}$ & $.64^{* * *}$ & $.33^{* * *}$ & $.36^{* * *}$ & $.33^{* * *}$ & $.29^{* * *}$ & $.34^{* * *}$ & - & $.73^{*}$ & $.70^{*}$ & 2.81 & 0.94 & .94 \\
\hline 12. Self-critical perfectionism & $.50^{* * *}$ & $.58^{* * *}$ & $.90^{*}$ & $.80^{* * *}$ & $.87^{* * *}$ & $.66^{* * *}$ & $.28^{* * *}$ & $.26^{* * *}$ & $.25^{* * *}$ & $.13^{*}$ & $.57^{* * *}$ & - & $.61^{*}$ & 2.86 & 1.03 & .95 \\
\hline 13. Narcissistic perfectionism & $.38^{* * *}$ & $.35^{* * *}$ & $.20^{*}$ & .06 & $.30^{* * *}$ & $.34^{* * *}$ & $.83^{*}$ & $.90^{*}$ & $.87^{* * *}$ & $.83^{* * *}$ & $.38^{*}$ & $.27^{* * *}$ & - & 2.31 & 0.88 & .96 \\
\hline$M$ & 3.02 & 3.02 & 3.08 & 3.12 & 2.94 & 2.57 & 2.20 & 1.85 & 1.89 & 2.03 & 3.02 & 2.94 & 1.99 & & & \\
\hline$S D$ & 0.92 & 0.95 & 0.99 & 0.91 & 1.02 & 0.87 & 0.95 & 0.87 & 0.89 & 0.92 & 0.90 & 1.02 & 0.78 & & & \\
\hline Cronbach's alpha $(\alpha)$ & .85 & .86 & .85 & .84 & .87 & .79 & .82 & .88 & .81 & .79 & .92 & .92 & .93 & & & \\
\hline
\end{tabular}

Note. Study $1(N=288)$ participants are below the diagonal; Study $2(N=352)$ participants are above the diagonal.

${ }^{*} p<.05 .{ }^{* *} p<.01 .{ }^{* * *} p<.001$. 


\section{BIG THREE PERFECTIONISM SCALE}

Table 2

Exploratory factor analysis of the unidimensionality of the BTPS facets

\begin{tabular}{|c|c|c|c|c|}
\hline Facets & No. of items & Eigenvalue 1 & Eigenvalue 2 & $\begin{array}{c}\text { Variance explained by } \\
\text { Eigenvalue } 1\end{array}$ \\
\hline Self-oriented perfectionism & 5 & 3.153 & 0.645 & 63.05 \\
\hline Self-worth contingencies & 5 & 3.216 & 0.538 & 64.32 \\
\hline Concern over mistakes & 5 & 3.094 & 0.574 & 61.89 \\
\hline Doubts about actions & 5 & 3.082 & 0.640 & 61.64 \\
\hline Self-criticism & 4 & 2.853 & 0.425 & 71.32 \\
\hline Socially-prescribed perfectionism & 4 & 2.457 & 0.629 & 61.42 \\
\hline Other-oriented perfectionism & 5 & 3.412 & 0.515 & 68.23 \\
\hline Hypercriticism & 4 & 2.581 & 0.542 & 64.54 \\
\hline Entitlement & 4 & 2.560 & 0.578 & 64.01 \\
\hline Grandiosity & 4 & 2.460 & 0.661 & 61.51 \\
\hline
\end{tabular}

Note. $N=288$. Factor extraction method $=$ maximum likelihood 


\section{BIG THREE PERFECTIONISM SCALE}

Table 3

BTPS: items and factor loadings from the exploratory and confirmatory factor analyses

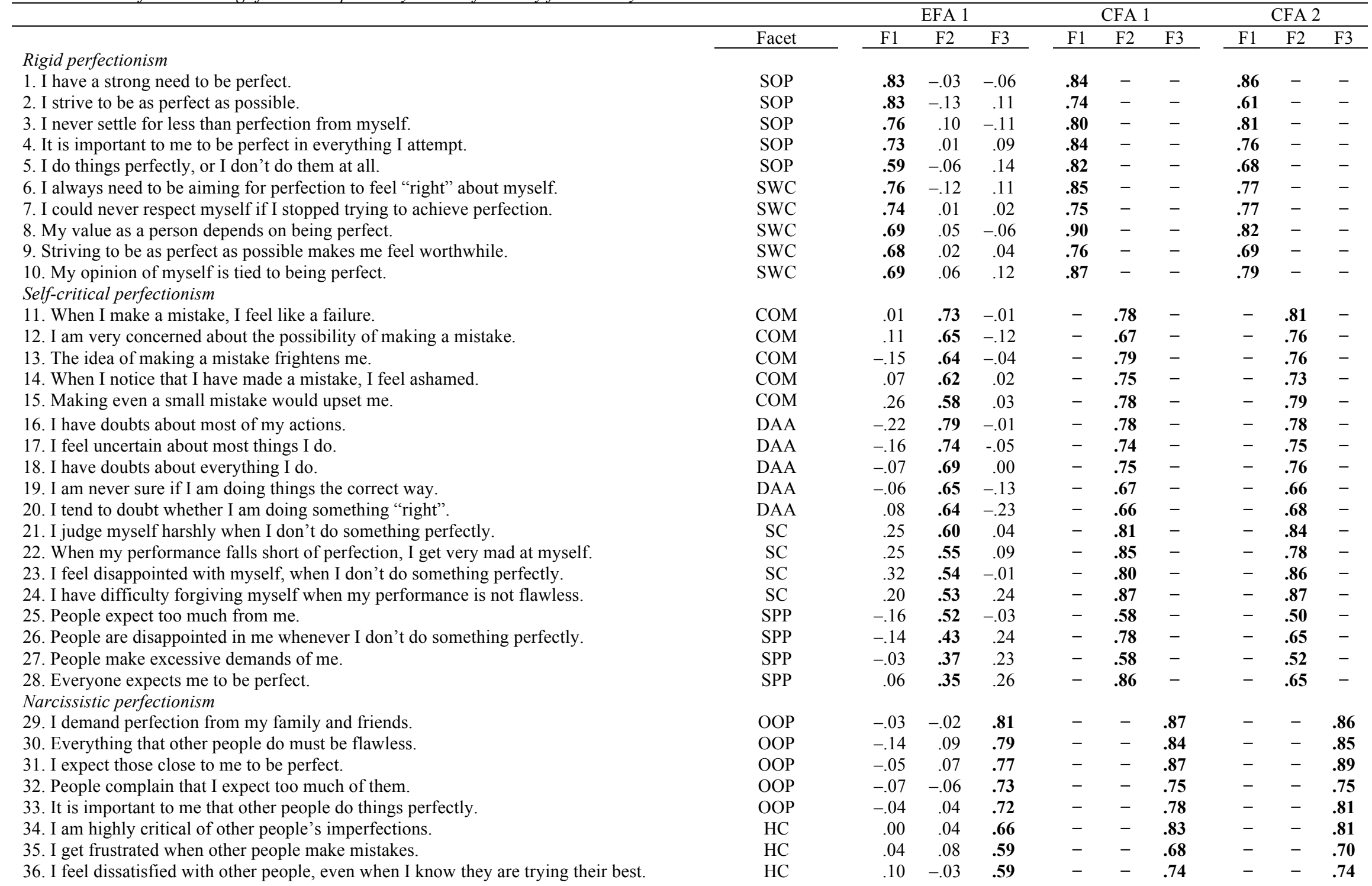




\section{BIG THREE PERFECTIONISM SCALE}

37. I am quick to point out other people's flaws.

38. I am entitled to special treatment.

39. I expect other people to bend the rules for me.

40. It bothers me when people don't notice how perfect I am.

41. I deserve to always have things go my way.

42. I am the absolute best at what I do.

43. I know that I am perfect.

44. Other people secretly admire my perfection.
$\mathrm{HC}$

ENT

ENT

ENT

ENT

GRAN

GRAN

GRAN

GRAN

\begin{tabular}{rrrrrrrrr}
-.01 & .12 & $\mathbf{. 5 6}$ & - & - & $\mathbf{. 7 2}$ & - & - & $\mathbf{. 7 1}$ \\
.03 & -.03 & $\mathbf{. 7 4}$ & - & - & $\mathbf{. 8 6}$ & - & - & .77 \\
-.10 & .07 & $\mathbf{. 6 8}$ & - & - & $\mathbf{. 8 1}$ & - & - & $\mathbf{7 1}$ \\
-.09 & .11 & $\mathbf{. 6 7}$ & - & - & $\mathbf{. 8 6}$ & - & - & $\mathbf{. 8 1}$ \\
.08 & -.18 & $\mathbf{. 6 5}$ & - & - & $\mathbf{. 8 2}$ & - & - & $\mathbf{. 7 6}$ \\
.06 & .01 & $\mathbf{. 6 3}$ & - & - & $\mathbf{. 6 9}$ & - & - & $\mathbf{. 7 3}$ \\
.07 & -.16 & $\mathbf{. 6 2}$ & - & - & $\mathbf{. 8 4}$ & - & - & $\mathbf{7 1}$ \\
.20 & -.11 & $\mathbf{. 5 9}$ & - & - & $\mathbf{. 8 5}$ & - & - & $\mathbf{. 7 5}$ \\
.18 & -.19 & $\mathbf{. 5 1}$ & - & - & $\mathbf{. 7 2}$ & - & - & $\mathbf{. 6 4}$ \\
\hline
\end{tabular}

Note. $\mathrm{F} 1=$ rigid perfectionism; $\mathrm{F} 2=$ self-critical perfectionism; F3 = narcissistic perfectionism. $\mathrm{SOP}=$ self-oriented perfectionism; $\mathrm{SWC}=$ self-worth contingencies, $\mathrm{COM}=$

concern over mistakes, DAA = doubts about actions; $\mathrm{SC}=$ self-criticism; $\mathrm{SPP}=$ socially prescribed perfectionism; OOP = other-oriented perfectionism; HC = hypercriticism; ENT $=$ entitlement; GRAN = grandiosity. EFA $(n=288)$ : Exploratory factor analysis with maximum likelihood estimation and promax rotation. CFA conducted using WLSMV estimation. 


\section{BIG THREE PERFECTIONISM SCALE}

Table 4

Second-order exploratory factor analysis of the BTPS facets

\begin{tabular}{|c|c|c|c|}
\hline \multirow[b]{2}{*}{ Facet } & \multicolumn{3}{|c|}{ Three-Factor Model } \\
\hline & $\begin{array}{c}\text { Rigid } \\
\text { perfectionism }\end{array}$ & $\begin{array}{c}\text { Self-critical } \\
\text { perfectionism }\end{array}$ & $\begin{array}{l}\text { Narcissistic } \\
\text { perfectionism }\end{array}$ \\
\hline Self-oriented perfectionism & .99 & -.07 & .01 \\
\hline Self-worth contingencies & .81 & .14 & .00 \\
\hline Concern over mistakes & .00 & .93 & -.04 \\
\hline Doubts about actions & -.06 & .74 & -.11 \\
\hline Self-criticism & .18 & .75 & .04 \\
\hline Socially-prescribed perfectionism & -.06 & .47 & .27 \\
\hline Other-oriented perfectionism & -.05 & .03 & .89 \\
\hline Hypercriticism & .03 & .07 & .72 \\
\hline Entitlement & -.09 & .05 & .86 \\
\hline Grandiosity & .15 & -.18 & .76 \\
\hline
\end{tabular}

Note. $N=288$. Pattern matrix. Factor extraction method $=$ maximum likelihood; rotation method $=$ promax. Loadings $>.30$ are boldfaced. $r$ (rigid perfectionism, self-critical perfectionism $)=.59, r($ rigid perfectionism, narcissistic perfectionism $)=.41, r($ self-critical perfectionism, narcissistic perfectionism $)=.28$. 


\section{BIG THREE PERFECTIONISM SCALE}

Table 5

Fit indices and factor loadings of unidimensional confirmatory factor analysis models of BTPS facets

\begin{tabular}{|c|c|c|c|c|c|}
\hline BTPS facets & $\chi^{2}$ & $d f$ & CFI & TLI & Loadings \\
\hline \multicolumn{6}{|l|}{ Study $2(N=352)$} \\
\hline Self-oriented perfectionism & 27.90 & 5 & .994 & .987 & .76 to .87 \\
\hline Self-worth contingencies & 63.22 & 5 & .984 & .968 & .72 to .89 \\
\hline Concern over mistakes & 11.84 & 5 & .997 & .995 & .76 to .83 \\
\hline Doubts about actions & 26.01 & 5 & .995 & .990 & .77 to .88 \\
\hline Self-criticism & 3.17 & 2 & 1.000 & .999 & .81 to .88 \\
\hline Socially-prescribed perfectionism & 47.58 & 2 & .975 & .926 & .77 to .88 \\
\hline Hypercriticism & 3.82 & 2 & .999 & .997 & .71 to .86 \\
\hline Other-oriented perfectionism & 2.96 & 5 & 1.000 & 1.000 & .77 to .90 \\
\hline Entitlement & 3.68 & 2 & 1.000 & .999 & .83 to .91 \\
\hline Grandiosity & 23.59 & 2 & .991 & .974 & .80 to .84 \\
\hline All facets & 1767.64 & 900 & .971 & .968 & .71 to .95 \\
\hline \multicolumn{6}{|l|}{ Study $3(N=290)$} \\
\hline Self-oriented perfectionism & 21.76 & 5 & .989 & .978 & .63 to .88 \\
\hline Self-worth contingencies & 18.75 & 5 & .990 & .980 & .71 to .86 \\
\hline Concern over mistakes & 6.94 & 5 & .999 & .999 & .76 to .82 \\
\hline Doubts about actions & 13.26 & 5 & .997 & .995 & .77 to .88 \\
\hline Self-criticism & 0.08 & 2 & 1.000 & 1.000 & .82 to .89 \\
\hline Socially-prescribed perfectionism & 30.75 & 2 & .976 & .929 & .78 to .80 \\
\hline Hypercriticism & 1.54 & 2 & 1.000 & 1.000 & .75 to .83 \\
\hline Other-oriented perfectionism & 7.98 & 5 & .998 & .997 & .75 to .91 \\
\hline Entitlement & 0.46 & 2 & 1.000 & 1.010 & .76 to .87 \\
\hline Grandiosity & 16.98 & 2 & .983 & .949 & .75 to .88 \\
\hline All facets & 1165.44 & 900 & .981 & .979 & .63 to .90 \\
\hline
\end{tabular}

Note. $\chi^{2}=$ weighted least squares estimation. 


\section{BIG THREE PERFECTIONISM SCALE}

Table 6

Study 3: Bivariate correlations between perfectionism indicator subscales scales and related measures

\begin{tabular}{|c|c|c|c|c|c|c|c|c|c|c|}
\hline Scale & SOP & SWC & $\mathrm{COM}$ & DAA & $\mathrm{SC}$ & SPP & OOP & $\mathrm{HC}$ & ENT & GRAN \\
\hline \multicolumn{11}{|l|}{ Perfectionism: FMPS } \\
\hline Concern over mistakes & $.51^{* * *}$ & $.56^{* * *}$ & $.66^{* * *}$ & $.48^{* * *}$ & $.69^{* * *}$ & $.53^{* * *}$ & $.43^{* * *}$ & $.41^{* * *}$ & $.31^{* * *}$ & $.25^{* * *}$ \\
\hline Doubts about actions & $.33^{* * *}$ & $.34^{* * *}$ & $.64^{* * *}$ & $.72^{* * *}$ & $.56^{* * *}$ & $.37^{* * *}$ & $.27^{* * *}$ & $.24^{* * *}$ & $.12^{*}$ & .08 \\
\hline Personal standards & $.61^{* * *}$ & $.56^{* * *}$ & $.43^{* * *}$ & $.20^{* * *}$ & $.56^{* * *}$ & $.32^{* * *}$ & $.26^{* * *}$ & $.27^{* * *}$ & $.20^{* * *}$ & $.26^{* * *}$ \\
\hline Parental criticism & $.16^{* *}$ & $.18^{* * *}$ & $.41^{* * *}$ & $.37^{* * *}$ & $.38^{* * *}$ & $.50^{* * *}$ & $.27^{* * *}$ & $.23^{* * *}$ & $.16^{*}$ & $.14^{*}$ \\
\hline Parental expectations & $.25^{* * *}$ & $.23^{* * *}$ & $.36^{* * *}$ & $.29^{* * *}$ & $.36^{* * *}$ & $.56^{* * *}$ & $.21^{* * *}$ & $.19^{* *}$ & .11 & $.12^{*}$ \\
\hline Organization & $.34^{* * *}$ & $.28^{* * *}$ & $.18^{* *}$ & .08 & $.17^{* *}$ & .01 & .00 & .00 & -.11 & .00 \\
\hline \multicolumn{11}{|l|}{ Perfectionism: HF-MPS } \\
\hline Self-oriented perfectionism & $.76^{* * *}$ & $.71^{* * *}$ & $.54^{* * *}$ & $.30^{* * *}$ & $.65^{* * *}$ & $.30^{* * *}$ & $.26^{* * *}$ & $.26^{* * *}$ & .08 & $.17^{*}$ \\
\hline Other-oriented perfectionism & $.30^{* * *}$ & $.32^{* * *}$ & $.18^{* * *}$ & .07 & $.26^{* * *}$ & $.19^{* * *}$ & $.52^{* * *}$ & $.53^{* * *}$ & $.33^{* * *}$ & $.29^{* * *}$ \\
\hline Socially prescribed perfectionism & $.35^{* * *}$ & $.35^{* * *}$ & $.49^{* * *}$ & $.37^{* * *}$ & $.47^{* * *}$ & $.69^{* * *}$ & $.28^{* * *}$ & $.30^{* * *}$ & $.17^{*}$ & $.16^{*}$ \\
\hline \multicolumn{11}{|l|}{ Five-factor personality } \\
\hline Neuroticism & $.18^{* * *}$ & $.23^{* * *}$ & $.40^{* * *}$ & $.36^{* * *}$ & $.34^{* * *}$ & $.15^{* * *}$ & $.31^{* * *}$ & $.39^{* * *}$ & $.22^{* * *}$ & .14 \\
\hline Openness & .10 & .11 & .01 & -.10 & .00 & .07 & .02 & .01 & .04 & .04 \\
\hline Conscientiousness & $.35^{* * *}$ & $.26^{* * *}$ & .04 & $-.13^{*}$ & .08 & -.06 & -.05 & -.08 & -.14 & .03 \\
\hline Agreeableness & -.05 & $-.15^{*}$ & $-.18^{* *}$ & $-.21^{* * *}$ & $-.17^{* *}$ & $-.15^{*}$ & $-.43^{* * *}$ & $-.55^{* * *}$ & $-.41^{* * *}$ & $-.29^{* * *}$ \\
\hline Extraversion & -.10 & -.08 & $-.37^{* * *}$ & $-.42^{* * *}$ & $-.33^{* * *}$ & -.11 & -.09 & $-.15^{* *}$ & -.01 & .00 \\
\hline
\end{tabular}

Note. $N=290$. SOP = self-oriented perfectionism; SWC = self-worth contingencies; COM = concern over mistakes; DAA = doubts about actions; SC = selfcriticism; OOP $=$ other-oriented perfectionism; HC $=$ hypercriticism; ENT $=$ entitlement; GRAN = grandiosity. FMPS = Frost Multidimensional Perfectionism Scale. HF-MPS = Hewitt-Flett Multidimensional Perfectionism Scale.

$* p<.05 . * * p<.01 . * * * p<.001$ 


\section{BIG THREE PERFECTIONISM SCALE}

Table 7

Study 3: Correlations between BTPS facets and global factors with perfectionism indicators and personality

\begin{tabular}{|c|c|c|c|c|c|c|}
\hline & \multicolumn{3}{|c|}{ Bivariate correlations } & \multicolumn{3}{|c|}{ Semipartial correlations } \\
\hline & $\begin{array}{c}\text { Rigid } \\
\text { perfectionism }\end{array}$ & $\begin{array}{c}\text { Self-critical } \\
\text { perfectionism }\end{array}$ & $\begin{array}{c}\text { Narcissistic } \\
\text { perfectionism }\end{array}$ & $\begin{array}{c}\text { Rigid } \\
\text { perfectionism }\end{array}$ & $\begin{array}{c}\text { Self-critical } \\
\text { perfectionism }\end{array}$ & $\begin{array}{c}\text { Narcissistic } \\
\text { perfectionism }\end{array}$ \\
\hline \multicolumn{7}{|l|}{ Perfectionism: FMPS } \\
\hline Concern Over Mistakes & $.56^{* * *}$ & $.71^{* * *}$ & $.41^{* * *}$ & $.21^{* * *}$ & $.55^{* * *}$ & $.17^{* * *}$ \\
\hline Doubts About Actions & $.35^{* * *}$ & $.71^{* * *}$ & $.21^{* * *}$ & -.05 & $.73^{* * *}$ & .01 \\
\hline Parental Expectations & $.25^{* * *}$ & $.46^{* * *}$ & $.19^{* *}$ & -.01 & $.45^{* * *}$ & .06 \\
\hline Personal Standards & $.61^{* * *}$ & $.45^{* * *}$ & $.29^{* * *}$ & $.51^{* * *}$ & $.16^{* *}$ & .06 \\
\hline Parental Criticism & $.18^{* *}$ & $.49^{* * *}$ & $.23^{* *}$ & $-.16^{* *}$ & $.54^{* * *}$ & $.13^{*}$ \\
\hline Organization & $.33^{* * *}$ & $.14^{*}$ & -.03 & $.39^{* * *}$ & -.02 & $-.16^{* *}$ \\
\hline \multicolumn{7}{|l|}{ Perfectionism: HF-MPS } \\
\hline Self-oriented perfectionism & $.77^{* * *}$ & $.54^{* * *}$ & $.23^{* * *}$ & $.69^{* * *}$ & $.19^{* * *}$ & -.07 \\
\hline Other-oriented perfectionism & $.32^{* * *}$ & $.21^{* * *}$ & $.49^{* * *}$ & $.19^{* *}$ & -.03 & $.44^{* * *}$ \\
\hline Socially prescribed perfectionism & $.36^{* * *}$ & $.58^{* * *}$ & $.27^{* * *}$ & .05 & $.52^{* * *}$ & .09 \\
\hline \multicolumn{7}{|l|}{ Five-factor personality } \\
\hline Neuroticism & $.22^{* * *}$ & $.39^{* * *}$ & $.31^{* * *}$ & -.05 & $.35^{* * *}$ & $.23^{* * *}$ \\
\hline Extraversion & -.10 & $-.39^{* * *}$ & -.08 & $.15^{*}$ & $-.47^{* * *}$ & .01 \\
\hline Openness & .11 & -.01 & .03 & $.17^{*}$ & -.11 & .00 \\
\hline Agreeableness & -.10 & $-.22^{* * *}$ & $-.50^{* * *}$ & $.15^{*}$ & $-.15^{*}$ & $-.50^{* * *}$ \\
\hline Conscientiousness & $.32^{* * *}$ & -.02 & -.07 & $.51^{* * *}$ & $-.24^{* * *}$ & $-.18^{* *}$ \\
\hline
\end{tabular}

Note. $N=287$. Semipartial correlations from multiple regression simultaneously entering rigid perfectionism, self-critical perfectionism, and narcissistic perfectionism. FMPS = Frost Multidimensional Perfectionism Scale. HF-MPS = Hewitt-Flett Multidimensional Perfectionism Scale.

$p<.05 .{ }^{* *} p<.01 .^{* * *} p<.001$ 


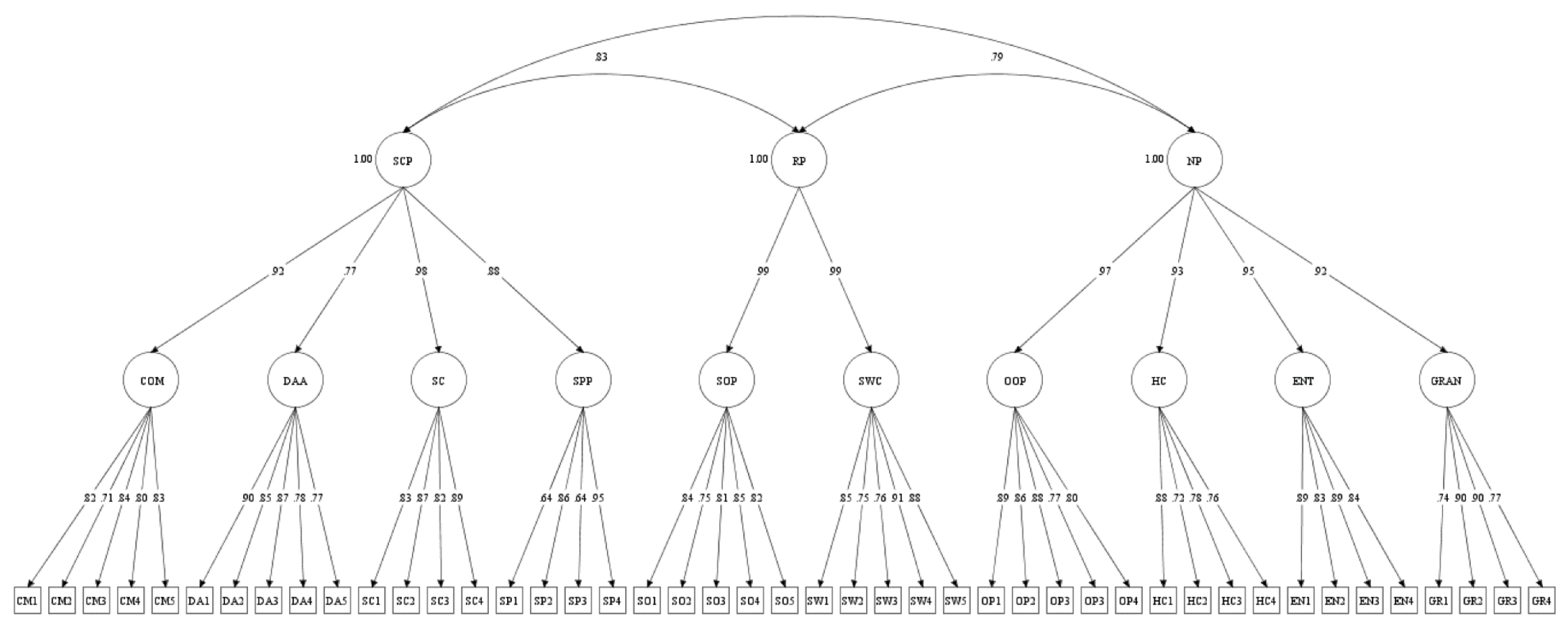

Figure 1. Study $2(N=352)$. Second-order factor analysis of the BTPS. SCP $=$ self-critical perfectionism; RP = rigid perfectionism; $\mathrm{NP}=$ narcissistic perfectionism; $\mathrm{COM}=$ concern over mistakes; DAA = doubts about actions; $\mathrm{SC}=$ self-criticism; $\mathrm{SPP}=$ socially-prescribed perfectionism; $\mathrm{SOP}=$ self-oriented perfectionism; $\mathrm{SWC}=$ self-worth contingencies; OOP = other-oriented perfectionism; $\mathrm{HC}=$ hypercriticism; $\mathrm{ENT}=$ entitlement; GRAN = grandiosity; CM1 to $\mathrm{CM} 4=$ concern over mistakes items 1 to 4 ; DA1 to DA5 = doubts about actions items 1 to 5 ; SC1 to SC4 = self-criticism items 1 to 4 ; SP1-SP4 = sociallyprescribed perfectionism items 1-4; SO1 to SO5 = self-oriented perfectionism items 1 to 5; SW1 to SW5 = self-worth contingencies items 1 to 5; OP1 to OP5 = other-oriented perfectionism items 1 to $5 ; \mathrm{HC} 1$ to $\mathrm{HC} 4=$ hypercriticism items 1 to 4 ; EN1 to EN4 = entitlement items 1 to 4; GR1 to GR $4=$ grandiosity items 1 to 4 . 
36

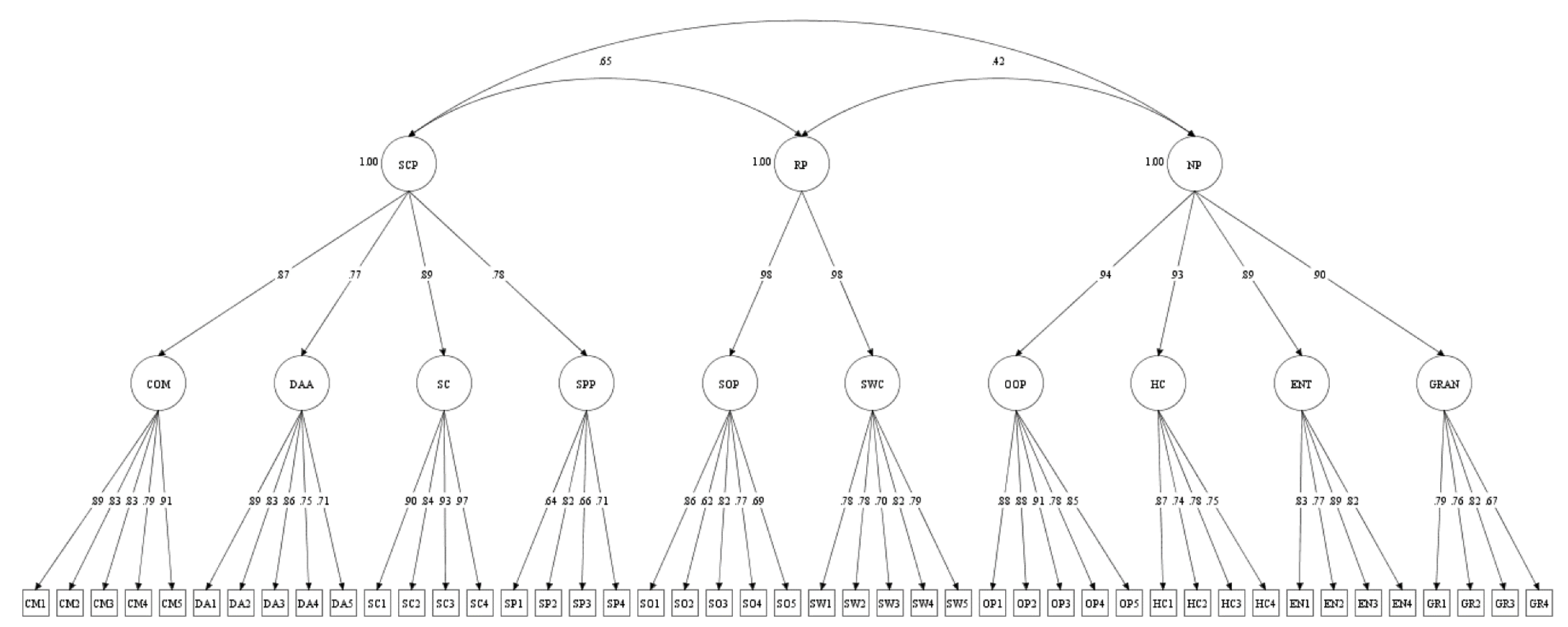

Figure 2. Study $3(N=290)$. Second-order factor analysis of the BTPS. SCP = self-critical perfectionism; RP = rigid perfectionism; $N P=$ narcissistic perfectionism; $\mathrm{COM}=$ concern over mistakes; $\mathrm{DAA}=$ doubts about actions; $\mathrm{SC}=$ self-criticism; $\mathrm{SPP}=$ socially-prescribed perfectionism; $\mathrm{SOP}=$ self-oriented perfectionism; SWC = self-worth contingencies; OOP = other-oriented perfectionism; HC = hypercriticism; ENT = entitlement; GRAN = grandiosity; CM1 to $\mathrm{CM} 4=$ concern over mistakes items 1 to 4 ; DA1 to DA5 = doubts about actions items 1 to 5 ; SC1 to SC4 = self-criticism items 1 to 4 ; SP1-SP4 = sociallyprescribed perfectionism items 1-4; SO1 to SO5 = self-oriented perfectionism items 1 to 5; SW 1 to SW5 = self-worth contingencies items 1 to 5 ; OP1 to OP5 = other-oriented perfectionism items 1 to $5 ; \mathrm{HC} 1$ to $\mathrm{HC} 4=$ hypercriticism items 1 to 4 ; EN1 to EN4 = entitlement items 1 to 4; GR1 to GR $4=$ grandiosity items 1 to 4 . 


\section{Supplementary Material \\ Big Three Perfectionism Scale (BTPS)}

Instructions: Please indicate how much you agree with each of the following statements.

\begin{tabular}{|c|c|c|c|c|}
\hline $\begin{array}{c}\text { Disagree } \\
\text { strongly }\end{array}$ & Disagree & $\begin{array}{c}\text { Neither agree } \\
\text { nor disagree }\end{array}$ & Agree & $\begin{array}{c}\text { Agree } \\
\text { strongly }\end{array}$ \\
\hline 1 & 2 & 3 & 4 & 5 \\
\hline
\end{tabular}

1. I have a strong need to be perfect. (SOP1)

2. I strive to be as perfect as possible. (SOP2)

3. I never settle for less than perfection from myself. (SOP3)

4. It is important to me to be perfect in everything I attempt. (SOP4)

5. I do things perfectly, or I don't do them at all. (SOP5)

6. I always need to be aiming for perfection to feel "right" about myself. (SWC1)

7. I could never respect myself if I stopped trying to achieve perfection. (SWC2)

8. My value as a person depends on being perfect. (SWC3)

9. Striving to be as perfect as possible makes me feel worthwhile. (SWC4)

10. My opinion of myself is tied to being perfect. (SWC5)

11. When I make a mistake, I feel like a failure. (COM1)

12. I am very concerned about the possibility of making a mistake. (COM2)

13. The idea of making a mistake frightens me. (COM3)

14. When I notice that I have made a mistake, I feel ashamed. (COM4)

15. Making even a small mistake would upset me. (COM5)

16. I have doubts about most of my actions. (DAA1)

17. I feel uncertain about most things I do. (DAA2)

18. I have doubts about everything I do. (DAA3)

19. I am never sure if I am doing things the correct way. (DAA4)

20. I tend to doubt whether I am doing something "right". (DAA5)

21. I judge myself harshly when I don't do something perfectly. (SC1)

22. When my performance falls short of perfection, I get very mad at myself. (SC2)

23. I feel disappointed with myself, when I don't do something perfectly. (SC3)

24. I have difficulty forgiving myself when my performance is not flawless. (SC4)

25. People expect too much from me. (SPP1)

26. People are disappointed in me whenever I don't do something perfectly. (SPP2)

27. People make excessive demands of me. (SPP3)

28. Everyone expects me to be perfect. (SPP4)

29. I demand perfection from my family and friends. (OOP1)

30. Everything that other people do must be flawless. (OOP2)

31. I expect those close to me to be perfect. (OOP3)

32. People complain that I expect too much of them. (OOP4)

33. It is important to me that other people do things perfectly. (OOP5)

34. I am highly critical of other people's imperfections. (HC1)

35. I get frustrated when other people make mistakes. (HC2)

36. I feel dissatisfied with other people, even when I know they are trying their best. (HC3)

37. I am quick to point out other people's flaws. (HC4)

38. I am entitled to special treatment. (ENT1) 
39. I expect other people to bend the rules for me. (ENT2)

40. It bothers me when people don't notice how perfect I am. (ENT3)

41. I deserve to always have things go my way. (ENT4)

42. I am the absolute best at what I do. (GRAN1)

43. I know that I am perfect. (GRAN2)

44. Other people secretly admire my perfection. (GRAN3)

45. Other people acknowledge my superior ability. (GRAN4)

Copyright by Martin M. Smith. All rights reserved. Permission granted to use and reproduce this measure for academic and research purposes.

\section{$\underline{\text { Scoring Directions for the BTPS }}$}

1. Self-oriented perfectionism (SOP; 5 items): Sum of items 1, 2, 3, 4, and 5

2. Self-worth contingencies (SWC; 5 items): Sum of items 6, 7, 8, 9, and 10

3. Concern over mistakes (COM; 5 items): Sum of items: 11, 12, 13, 14, and 15

4. Doubts about action (DAA; 5 items): Sum of items: 16, 17, 18, 19, 20

5. Self-criticism (SC; 4 items): Sum of items: 21, 22, 23, and 24

6. Socially-prescribed perfectionism (SPP; 4 items): Sum of items: 25, 26, 27, and 28

7. Other-oriented perfectionism (OOP; 5 items): Sum of items: 29, 30, 31, 32, and 33

8. Hypercriticism (HC; 4 items): Sum of items: 34, 35, 36, and 37

9. Entitlement (ENT: 4 Items): Sum of items: 38, 39, 40, and 41

10. Grandiosity (GRAN: 4 Items): Sum of items 42, 43, 44, and 45

11. Rigid perfectionism: Sum of self-oriented perfectionism and self-worth contingencies.

12. Self-critical perfectionism: Sum of concern over mistakes, doubts about actions, selfcriticism, and socially-prescribed perfectionism.

13. Narcissistic Perfectionism: Sum of other-oriented perfectionism, hypercriticism, entitlement, and grandiosity. 